\section{STM Manipulation of Single Molecules Provides a Route to Molecular Switching}

For the past few years, research teams have investigated the electronic properties and the interaction of molecules with metallic surfaces using a scanning tunneling microscope (STM) and the synthesis of molecules for nanoscale experiments. The molecules manipulated by the STM tip must exhibit electronic effects such as switching in order to be useful in the creation of nanoscale devices. An international team of researchers affiliated with the Institut für Experimentalphysik; Freie University, Berlin; Paul Drude Institut für Festkörperelektronik, Hausvogteiplatz, Germany; and CEMES-CNRS, France, has reported results on experimental and theoretical work regarding the STM manipulation of individual porphyrin-based molecules and the related phenomena.

As reported in the January 22 issue of Physical Review Letters, the researchers used Cu-TBPP (Cu-tetra-3,5 di-ter-butyl-phenyl porphyrin) molecules evaporated on a $\mathrm{Cu}(211)$ surface (prepared in several steps with nanofacets and terraces by Ne sputtering and annealing to $700 \mathrm{~K})$. Performing calculations by the elastic scattering quantum chemistry technique for 770 molecular orbitals, they found that the electrical resistance $R$ of the Cu-TBPP molecules varies drastically by a $90^{\circ}$ rotation of one leg of the molecule. Hence, by using STM, it was shown that the internal conformation of the molecule can be modified reversibly, as well as the electric properties of the molecules. Two distinct situations were emphasized, corresponding to two different phases of the molecule: The tunneling resistance through a leg decreases for the flat case, compared with the rotated case, by one order of magnitude. The two situations were called "OFF-state" and "ON-state," respectively. The researchers also observed that the switching occurs for lateral and vertical manipulation conditions as well. Experimentally, the orientation of the leg can be switched using the STM tip, causing a current variation dependent on the distance between the tip and that molecule that is in very good agreement with theoretical predictions. The conductance differs by two orders of magnitude between the $\mathrm{ON}$ and OFF states of the molecule, and the switching process is fully reversible.

With this work, the researchers have shown that by means of vertical and lateral manipulation, it is possible to rotate single legs around their $\sigma$-bond, in and out of the porphyrin plane, and that the controlled rotation of the legs induced by the STM tip realizes the principle of a conformational molecular switch.

Claudiu Muntele

\section{Pseudopotential Calculations Explain the Structure and Electrical Conductance of Single-Walled Carbon Nanotubes}

Carbon nanotubes have drawn the interest of many researchers because of their extremely strong mechanical properties and adjustable electrical properties. Recently, researchers at the University of California-Berkeley and Seoul National University, South Korea, have carried out first-principles calculations to investigate the structural deformation and intertube conductance of crossed carbon nanotube junctions. Each of these junctions consists of two $(5,5)$ single-walled nanotubes (SWNT) crossing each other.

As reported in the January 22 issue of Physical Review Letters, the researchers employed an $a b$ initio pseudopotential density functional approach in linear combination of atomic orbitals (LCAO) basis. They considered a resistive junction region and four perfect regions (metallic leads) composed of semi-infinite perfect carbon nanotubes, where the junction is modeled to consist of two $(5,5)$ SWNTs crossing at a $90^{\circ}$ angle. The resulting inhomogeneous linear equations for scattering states were solved to obtain the transmission coefficients, which were then used to compute the conductance within the Landauer-Büttiker formalism.

The junction's geometrical structure was determined using a constrained totalenergy minimization, in which the position of the atoms near the junction was fully relaxed while fixing the center-tocenter intertube distance.
"The effect of nanotube-substrate interaction," according to Steven G. Louie, a professor from UC-Berkeley, "is modeled by a resulting contact force at the junction." Calculation shows that the contact distance-the shortest distance between atoms at different tubes-is a steep function of contact force between the two crossed SWNTs only in the range of 1-10 $\mathrm{nN}$. The distance saturates near $2.5 \AA$, and a further increase of the force would deform the tube shape.

The linear-response conductance of the crossed-tube junction was computed after the structure was known. The intratube and intertube conductance were computed as a function of the incident electron's energy for a 4-terminal device in a 4-terminal 4-probe measurement setup.

The researchers found that junction deformation plays an important role in the thus in the intertube conductance. For moderate contact forces, a smaller contact distance corresponds to a larger intertube conductance. Their calculation results also explain the recent experimental data on this system and suggest that this system can be employed for molecular-scale electromechanical devices.

WIRAWAN PURWANTO

\section{Six-Sided Flat Carbon \\ Molecules Theorized}

Paul von Ragué Schleyer, a computational chemist at the University of Georgia, has discovered that hexacoordinate, or six-sided flat carbon molecules, are theoretically possible. While carbon intratube and intertube transmissions, and

\section{Review Articles}

The November 24, 2000, issue of Science contains the following review articles on Issues in Nanotechnology: H.G. Craighead, "Nanoelectromechanical Systems"; S.R. Quake and A. Scherer, "From Micro- to Nanofabrication with Soft Materials"; and E.W.H. Jager, E. Smela, and O. Inganäs, "Microfabricating Conjugated Polymer Actuators."

The January 2001 issue of Reviews of Scientific Instruments contains a review article by I. Walmsley, L. Waxer, and C. Dorrer on "The Role of Dispersion in Ultrafast Optics."

The January 2001 issue of the Journal of Vacuum Science $\mathcal{E}$ Technology A contains a review article by A. Keller, M. Fierz, K. Siegmann, H.C. Siegmann, and A. Filippov on "Surface Science with Nano-sized Particles in a Carrier Gas."

The January 2001 issue of Reviews of Modern Physics contains the following review articles: P.B. Weichman, A.W. Harter, and D.L. Goodstein, "Criticality and Superfluidity in Liquid "He under Nonequilibrium Conditions"; A. Rousse, C. Rischel, and J.-C. Gauthier, "Femtosecond X-Ray Crystallography"; and W.M.C. Foulkes, L. Mitas, R.J. Needs, and G. Rajagopal, "Quantum Monte Carlo Simulations of Solids."

The January 2001 issue of Semiconductors contains a review article by I.M. Vikulin, L.F. Vikulina, and V.I. Stafeev on "Magnetotransistors."

The December 2000 issue of Physics of Atomic Nuclei contains a review article by V.T. Voronchev and V.I. Kukulin on "Nuclear-Physics Aspects of Controlled Thermonuclear Fusion: Analysis of Promising Fuels and Gamma-Ray Diagnostics of Hot Plasma." 